\title{
Growing up with cerebral palsy: What are the concerns for mental health?
}

\section{Laura Brunton}

\section{University of Western Ontario}

Cerebral palsy is a movement disorder accompanied by weakness and a myriad of associated secondary conditions including joint deformities, pain and fatigue; and is traditionally regarded as a pediatric neurological disorder. ${ }^{1}$ However, the majority of children with cerebral palsy will inevitably become adults with cerebral palsy ${ }^{3}$ and transition into a healthcare model with little focus on their specific needs, including mental health concerns. There is large variability in the motor impairments related to cerebral palsy. ${ }^{2}$ Related to the wide severity spectrum associated with this condition, individuals with cerebral palsy need varying degrees of support in a number of areas including physical, social, and psychological.

The period between childhood and adult life is particularly important for individuals with cerebral palsy. Adolescence is a trying time for any individual; however, adding a physical disability and the process of learning to self-manage their own healthcare can add further stress. ${ }^{4}$ There is a great deal of literature regarding parental stress in caregivers of children with cerebral palsy; yet there is a profound lack of information about the impact of cerebral palsy on the mental health of the child or teen. If parents of these children are feeling the stress of navigating the healthcare needs associated with cerebral palsy, it is not unrealistic to expect a similar impact on the adolescent when first beginning to take responsibility for their own care.

The relationship between lack of participation in meaningful activities and mental health issues, including depression and anxiety, has not yet been explored in adolescents with cerebral palsy specifically. However, children with chronic health conditions are consistently concerned with developing and maintaining friendships, and increased social isolation can have a negative effect on their quality of life. ${ }^{4}$ Adolescents with disabilities have the same dreams and aspirations for adult life as all young people; ${ }^{5}$ however, they can be restricted in their opportunities to practice or develop the life skills required to achieve specific goals and participate in meaningful activities. Often the lack of available support and resources to develop these skills can lead to further social isolation and withdrawal from community participation. ${ }^{6}$ It is unknown if children and adolescents with cerebral palsy experience the same mental health concerns described in other chronic health conditions, or if the specific diagnosis and physical manifestation of cerebral palsy has unique characteristics related to mental health. However, there are increased rates of depression and psychological difficulties experienced by children and adults with cerebral palsy compared to their age-matched peers. ${ }^{7,8}$

The nature of the relationship between common physical symptoms of cerebral palsy and mental health concerns has not yet been clearly described and warrants further study. However, in an ongoing study, several individuals with cerebral palsy have described a reciprocal relationship between stress and fatigue whereby increased stress leads to further exacerbation of physical symptoms and vice versa (Brunton \& Bartlett, unpublished data). This relationship needs to be studied directly to understand the impact of mental health issues on the physical symptoms of the condition.

Perrin and colleagues ${ }^{4}$ have suggested that the severity of the impairment and the presence of pain can influence or intensify the psychological impact of chronic conditions during adolescence. Chronic pain and fatigue are common physical symptoms of cerebral palsy and may intensify any underlying mental health concerns as individuals with cerebral palsy become adults. In a recent study, van der Slot and colleagues demonstrated a relationship between severity of fatigue and depressive symptoms in adults; however, the strength and impact of this relationship during the adolescent years is still unknown. ${ }^{8}$ Similarly, another study has demonstrated significant 
moderate correlations between the number of painful sites and mental fatigue in non-ambulatory adults with cerebral palsy. ${ }^{9}$ In light of this emerging correlational evidence there is a need for further investigation into the relationships that exist between psychological and physiological symptoms, in addition to understanding the basic psychological impact of living with cerebral palsy.

There is a dearth of information about the mental health concerns of individuals with cerebral palsy across all stages of life. The information available highlights the need to explore the mental health concerns adolescents with cerebral palsy face during late childhood through the transition years and into early adulthood, particularly since adolescence comes with additional stresses (including learning to self-manage their condition and coping with social isolation). Uncertainty and unpredictability experienced by individuals with cerebral palsy may cause increased psychological stress and potentially increase the expression of physical symptoms associated with cerebral palsy. Further information is needed about the health concerns related to depression, anxiety and stress experienced by adolescents with cerebral palsy. Clinicians need more information to assist with strategies and specific intervention planning to help these individuals cope with and anticipate the demands of transitioning to adult life.

\section{References}

1. Hilberink SR, Roebroeck ME, Nieuwstraten W, Jalink L, Verheijden J, Stam $\mathrm{HJ}$. Health issues in young adults with cerebral palsy: towards a life-span perspective. J Rehabil Med 2007;39(8):605-611.

2. Palisano R, Rosenbaum P, Walter S, Russell D, Wood E, Galuppi B. Development and reliability of a system to classify gross motor function in children with cerebral palsy. Dev Med Child Neurol 2008;39(4):214-223.

3. Colver A. Outcomes for people with cerebral palsy: life expectancy and quality of life. Paediatr Child Health 2012;22(9):384-387.

4. Perrin J, Gnanasekaran S, Delahaye J. Psychological Aspects of Chronic Health Conditions. Pediatr Rev 2012;33(3):99-109.

5. King GA, Baldwin PJ, Currie M, Evans J. Planning successful transitions from school to adult roles for youth with disabilities. Child Health Care 2005;34(3):193-216.

6. Blackorby J, Wagner M. Longitudinal postschool outcomes of youth with disabilities: Findings from the National Longitudinal Transition Study. Except Child 1996;62:399-414.

7. Lollar DJ. Encouraging personal and interpersonal independence. In: Lollar DJ, ed. Preventing Secondary Conditions Associated with Spina Bipda or Cerebral Palsy. Washington, DC: Spina Bifida Association of America; 1994: 17-25.

8. van der slot $W$, Nieuwenhuijsen $C$, van den Berg-Emons $R$, Bergen $M$, Hilberink SR, Stam HJ, et al. Chronic pain, fatigue, and depressive symptoms in adults with spastic bilateral cerebral palsy. Dev Med Child Neurol 2012;54(11):1064

9. Malone LA, Vogtle LK. Pain and fatigue consistency in adults with cerebral palsy. Disabil Rehabil 2010;32(5):385-391.

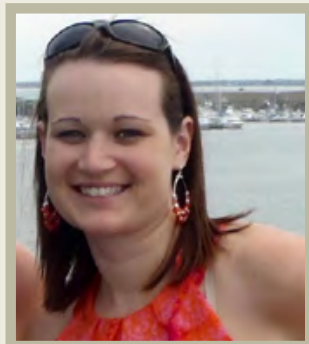

\section{Laura Brunton}

Laura completed her MSc at Western University under the supervision of Dr. Doreen Bartlett. Her master's research centered on determining the validation, reliability, responsiveness and sensitivityto-change of two abbreviated versions of the Gross Motor Function Measure (GMFM-66) for children with cerebral palsy. Laura is currently in a combined PhD/Master's of Physical Therapy program at Western University and her current research focus is understanding and measuring fatigue in adolescents and young adults with cerebral palsy. 